\title{
miR-124 Regulates Diverse Aspects of Rhythmic Behavior in Drosophila
}

\author{
Daniel L. Garaulet, ${ }^{1 *}$ Kailiang Sun, ${ }^{1,2 *}$ Wanhe Li, ${ }^{3 *}$ Jiayu Wen, ${ }^{1}$ Alexandra M. Panzarino, ${ }^{1}$ Jenna L. 0'Neil, ${ }^{3}$ \\ P. Robin Hiesinger, ${ }^{4}$ Michael W. Young, ${ }^{3}$ and Eric C. Lai ${ }^{1}$ \\ ${ }^{1}$ Sloan-Kettering Institute, Department of Developmental Biology, New York, New York 10065, ${ }^{2}$ Neuroscience Program, Weill Graduate School of Medical \\ Sciences, Cornell University, New York, New York 10065, ${ }^{3}$ Laboratory of Genetics, The Rockefeller University, New York, New York 10065, and ${ }^{4}$ Institute \\ for Biology, Freie Universitaet Berlin, 14195 Berlin, Germany
}

Circadian clocks enable organisms to anticipate and adapt to fluctuating environmental conditions. Despite substantial knowledge of central clock machineries, we have less understanding of how the central clock's behavioral outputs are regulated. Here, we identify Drosophila miR-124 as a critical regulator of diurnal activity. During normal light/dark cycles, mir-124 mutants exhibit profoundly abnormal locomotor activity profiles, including loss of anticipatory capacities at morning and evening transitions. Moreover, mir-124 mutants exhibited striking behavioral alterations in constant darkness (DD), including a temporal advance in peak activity. Nevertheless, anatomical and functional tests demonstrate a normal circadian pacemaker in mir-124 mutants, indicating this miRNA regulates clock output. Among the extensive miR-124 target network, heterozygosity for targets in the BMP pathway substantially corrected the evening activity phase shift in DD. Thus, excess BMP signaling drives specific circadian behavioral output defects in mir-124 knock-outs.

Key words: activity mode; activity phase; BMP signaling; circadian rhythm; Drosophila; microRNA

\section{Significance Statement}

Circadian clocks control rhythmic behaviors of most life-forms. Despite extensive knowledge of the central clock, there is less understanding of how its behavioral outputs are regulated. Here, we identify a conserved neural microRNA as a critical regulator of diurnal behavior. We find Drosophila mir-124 mutants exhibit robust activity abnormalities during normal light/dark cycles and during constant darkness. Nevertheless, as the central pacemaker is functional in these mutants, miR-124 regulates clock output. We provide mechanistic insight by showing deregulation of miR-124 targets in BMP signaling drives specific mir-124 defects. In summary, Drosophila mir-124 mutants reveal post-transcriptional control of circadian activities, and impact of BMP signaling in behavioral output.

\section{Introduction}

Most life-forms are behaviorally tuned to $24 \mathrm{~h}$ day/night cycles by an internal circadian clock. Molecular and neuronal bases of the clock are particularly well studied in Drosophila. At the core of the molecular clock, heterodimeric transcription fac-

Received Sept. 1, 2015; revised Jan. 21, 2016; accepted Jan. 22, 2016.

Author contributions: K.S., D.L.G., W.L., and E.C.L. designed research; K.S., D.L.G., W.L., P.R.H., A.M.P., and J.L.O. performed research; K.S., D.L.G., W.L., J.W., P.R.H., M.W.Y., and E.C.L. analyzed data; K.S. and E.C.L. wrote the paper.

This work was supported by the National Institutes of Health (Grant NIH-R01-EY018884 to P.R.H.; Grants NIHR01-GM083300, NIH-R01-NS074037, and R01-NS083833 to E.C.L.) and by the Memorial Sloan Kettering Cancer Center (Core Grant P30-CA008748 to E.C.L.). W.L. was supported by fellowships from the Jane Coffin Childs Memorial Fund for Medical Research and the Leon Levy Foundation. Work in M.W.Y.'s group was supported by NIH-NSO53087. We thank Dong Wang for helping with ERGs, and Yong Zhang and Patrick Emery for discussing unpublished data. We thank the Bloomington Stock Center, Developmental Studies Hybridoma Bank, Steve Cohen, Pejmun Haghighi, and Hiroki Ueda for reagents.

The authors declare no competing financial interests.

*D.L.G., K.S., and W.L. contributed equally to this work.

Correspondence should be addressed to Eric C. Lai at the above address. E-mail: laie@mskcc.org.

DOI:10.1523/JNEUROSCI.3287-15.2016

Copyright $\odot 2016$ the authors $\quad 0270-6474 / 16 / 363414-08 \$ 15.00 / 0$ tors CLOCK and CYCLE activate period (per) and timeless ( tim), whose products feed back to repress CLOCK/CYCLE activity (Allada and Chung, 2010). Post-translational regulators, such as kinases and phosphatases, can adjust feedback inhibition delay, and hence oscillator length. This negative feedback loop represents a self-sustaining mechanism that can direct rhythmic neuronal activity and behavior, in the absence of external cues. However, inputs, including light and temperature, help synchronize internal clocks to the environmental 24 h cycle (Busza et al., 2007).

Locomotor rhythmicity in Drosophila is controlled by $\sim 150$ circadian neurons in the central brain, organized into distinct subgroups (Helfrich-Förster, 2005). Under laboratory light/dark (LD) cycles, fruit flies exhibit bimodal activity with morning and evening peaks (Grima et al., 2004; Stoleru et al., 2004). Small ventral lateral neurons (s-LNvs) expressing pigment-dispersing factor (PDF) are necessary and sufficient for morning activity and termed morning cells ( $\mathrm{M}$ cells), whereas evening activity depends on dorsal lateral neurons (LNds) and possibly a subset of dorsal 

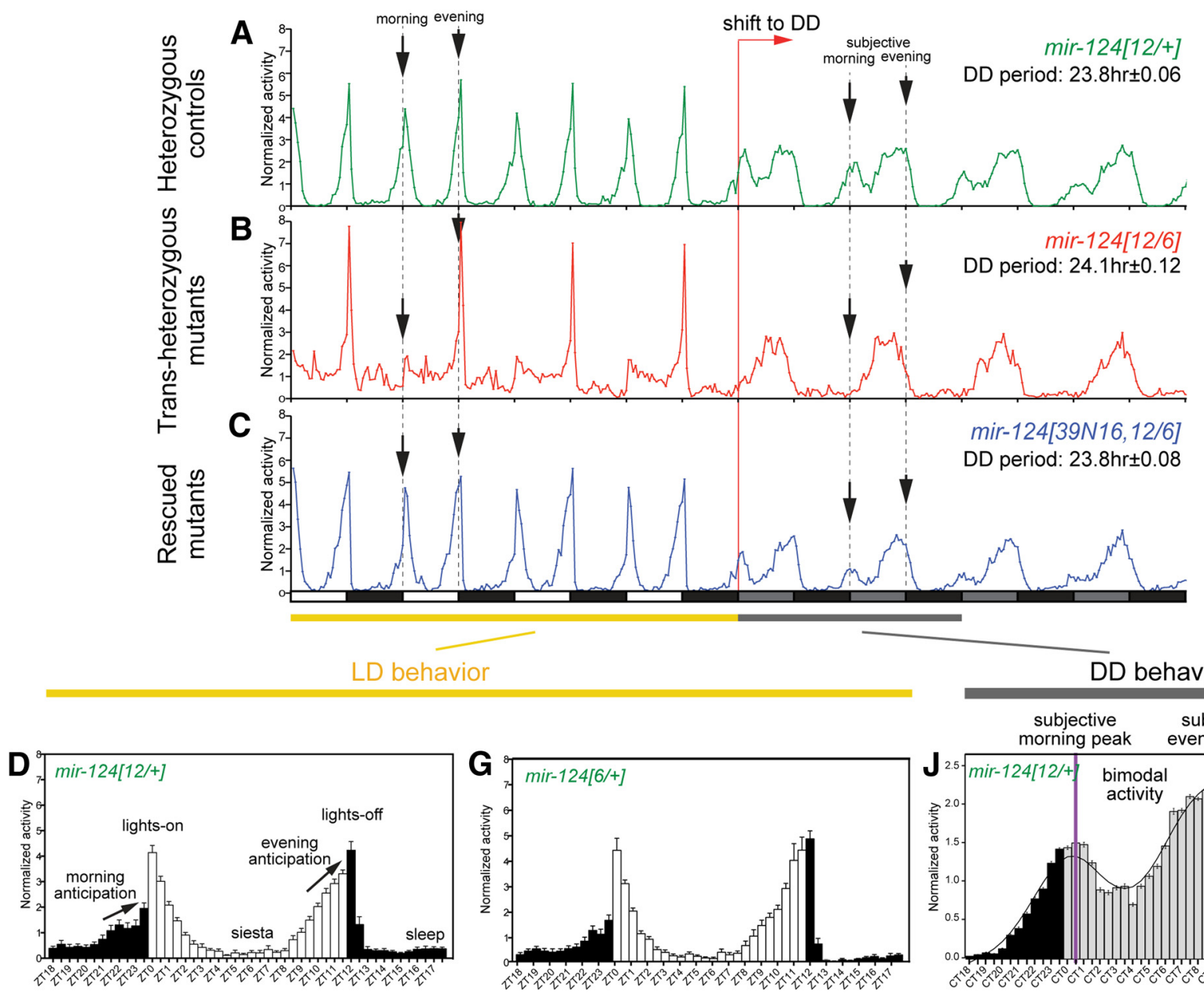

DD behavior
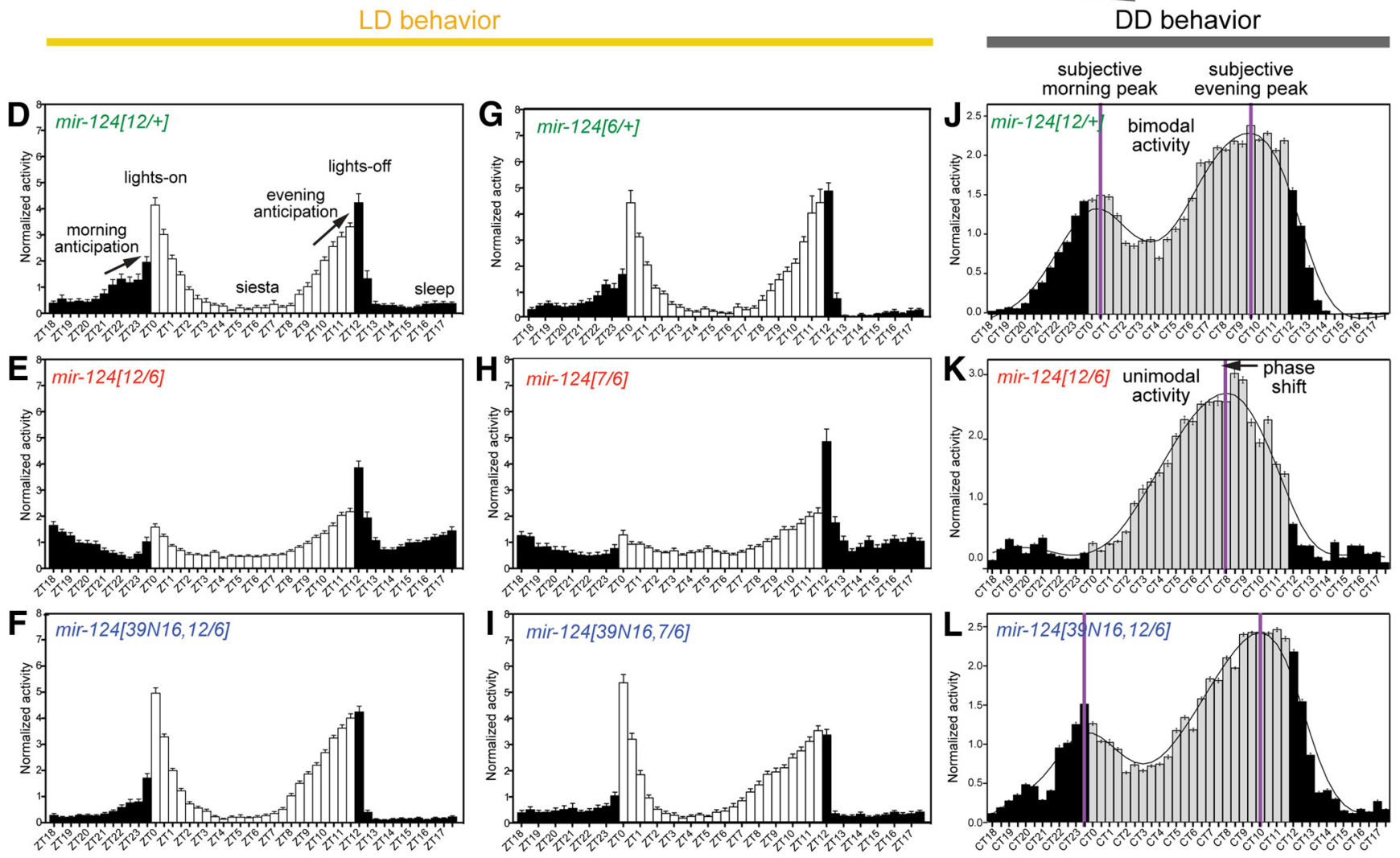

Figure 1. mir-124 knock-out impairs circadian behavior in LD and DD conditions. Panels depict male mir-124 heterozygous controls or trans-heterozygous mutants, or rescues bearing a $19 \mathrm{~kb}$ genomic transgene [39N16].A-C, Averaged activity profiles across multiple $12 \mathrm{~h} \mathrm{LD}$ cycles followed by DD. White bars represent light phase (day); black bars represent dark phase (night); subjective daytime during DD is represented in gray. Representative arrows and arrowheads indicate morning and evening peaks, respectively. The activity peaks in mir-124 mutants correspond to startle responses to light changes (see also $\boldsymbol{D}, \boldsymbol{E}$ ); dotted lines provide temporal register showing they occur after the anticipatory behaviors seen in control and rescued mutants. Note that despite their altered activity profile, mir- 124 mutants maintain rhythmicity in DD; period lengths are quantified. D-I, Averaged activity profiles across five LD cycles; error bars indicate SEM. Heterozygotes exhibit biphasic activity profile peaking around morning and evening transitions, whereas mir-124 mutants exhibit flattened behavior profiles; the defects are rescuable. $J-L$, Averaged activity profiles during the first two DD cycles following transfer to DD. Control mir-124 heterozygotes exhibit a bimodal DD activity profile with peaks around subjective morning and evening. Loss of miR-124 induces a unimodal profile in which evening peak activity is advanced. These behavioral defects are rescuable.

neuron 1 cells [DN1s; collectively termed evening cells (E cells); Grima et al., 2004; Stoleru et al., 2004]. In DD, s-LNvs serve as master pacemakers that generate circadian rhythm and dictate the period and phase of E cells (Stoleru et al., 2005).
Beyond transcriptional and post-translational strategies, evidence is emerging for post-transcriptional clock regulators (Lim and Allada, 2013). As key regulators of mRNA stability and translation, microRNAs (miRNAs) are implicated in multiple aspects of time 

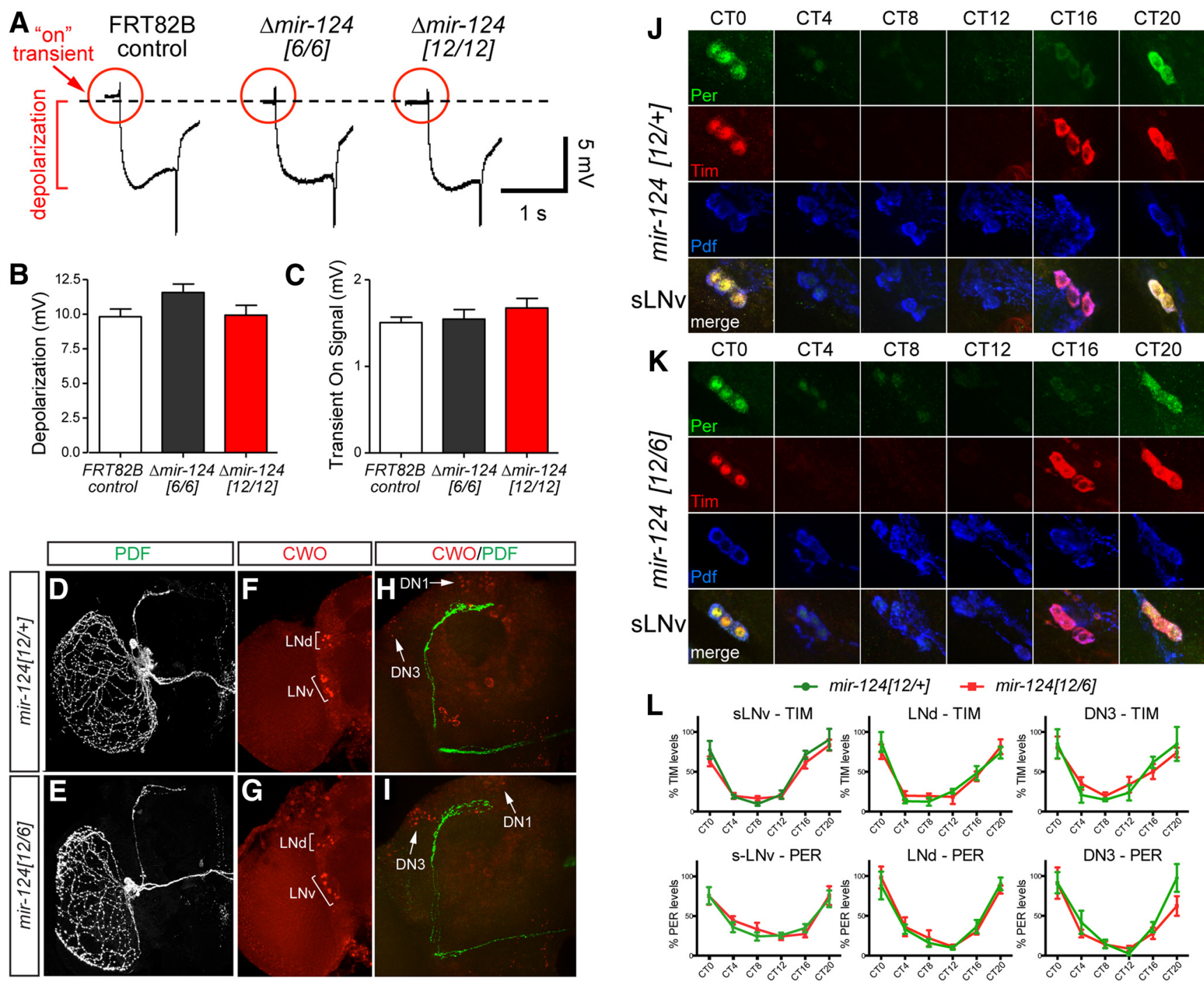

Figure 2. Normal phototransduction and core circadian cell function in mir-124 mutants. $\boldsymbol{A}$, Representative ERG traces for $1 \mathrm{~s}$ light stimulations shows normal activity profiles in the absence of miR-124. $\boldsymbol{B}$, Depolarization is a measure of how strongly photoreceptor neurons respond to the light stimulus. $\boldsymbol{C}$, "On" transients $(\boldsymbol{A}$, red circles) reveal the response of postsynaptic neurons and are therefore a measure for neurotransmission. All electrophysiological responses are normal in mir-124 mutants. D-I, Labeling of M and E cells by PDF and CW0 antibodies in heterozygous and mutant brains. PDF is expressed only in LNv neurons, which extend dendrites to the retina and send axon projections to the medial brain. CWO marks nuclei of LNv and LNd neurons, as well as certain dorsal neurons. No substantial specification or projection defects were observed in mir-124 mutants. $J, \boldsymbol{K}$, Confocal imaging of sLNvs immunostained for PER, TIM, and PDF in heterozygous and mutant brains dissected at indicated time points. $\boldsymbol{L}$, Quantification of relative PER and TIM levels across the indicated circadian time points. Error bars indicate $S E M$ in $\boldsymbol{B}, \boldsymbol{C}$, and $\boldsymbol{L}$.

keeping. miR-219 and miR-132 were early miRNAs identified to regulate mammalian clocks (Cheng et al., 2007). miR-219 is transcriptionally activated by CLOCK to affect clock pace, whereas CREB-regulated miR-132 attenuates light-induced clock resetting (Cheng et al., 2007; Alvarez-Saavedra et al., 2011). In Drosophila, knockdown of dicer-1 in circadian tissues dampened locomotor rhythmicity, and Clock is repressed by bantam miRNA (Kadener et al., 2009). In addition, knockdown of the miRNA effector GW182 caused circadian defects via defective PDF signaling (Zhang and Emery, 2013). Individual fly miRNA knock-outs, including let-7 (Chen et al., 2014), mir-959 964 (Vodala et al., 2012), and mir-279/mir996 (Luo and Sehgal, 2012; Sun et al., 2015b), affect various aspects of rhythmic behavior.

Here, we study conserved neural miR-124 in regulation of diurnal behavior. Deletion of Drosophila miR-124 severely disrupts activity profiles during LD cycles and in DD. As core circadian functions are maintained, miR-124 regulates clock output. Notably, we demonstrate that circadian phase advance in mir-124 mutants functionally involves derepression of miR-124 targets in BMP signaling. Together, these analyses provide insight into post-transcriptional control of circadian behaviors.

\section{Materials and Methods}

Drosophila genetics. Mutant and rescue alleles for mir-124 and UASDsRed-mir-124 have been described (Sun et al., 2012; Weng and Cohen, 2012). $D f(2 L)$ Exel7069 was from the Bloomington Stock Center. Circadian drivers pdf-Gal4, cry13-Gal4, and tim-Gal4 were maintained by the Young Laboratory (Rockefeller University). BMP pathway mutants were from Pejmun Haghighi (McGill University).

Electroretinograms. Electroretinograms (ERGs) were performed with these parameters. We used $2 \mathrm{M} \mathrm{NaCl}$ in the recording and reference electrodes. Electrode voltage was amplified by a Digidata 1440A, filtered through a Warner IE-210, and recorded using Clampex 10.1 by Molecular Devices. A postrecording filter was also provided by the Clampex software. Light stimulus was provided in $1 \mathrm{~s}$ pulses by a computer-controlled white LED system (Schott MC1500). 
A

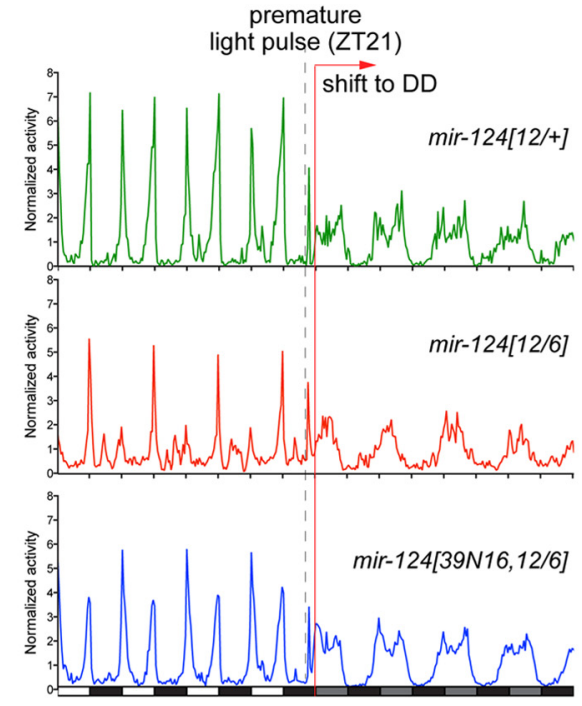

C
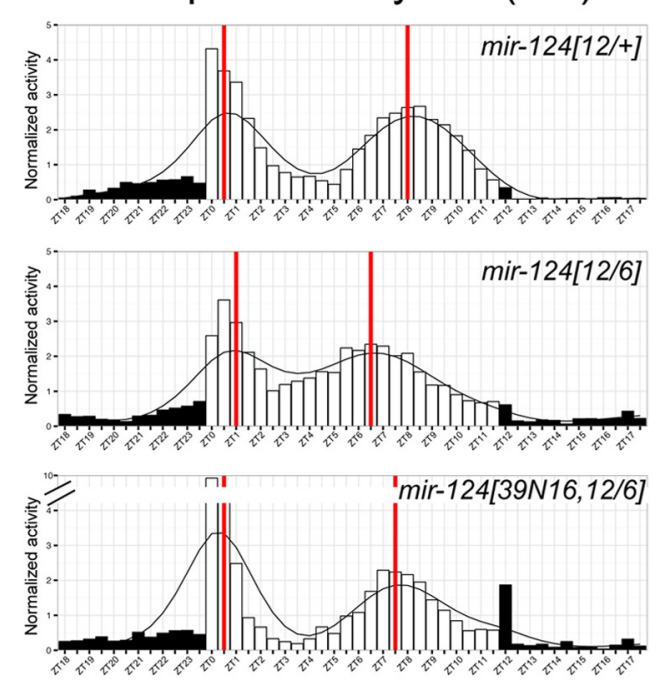

E Constant Temperature (DD)
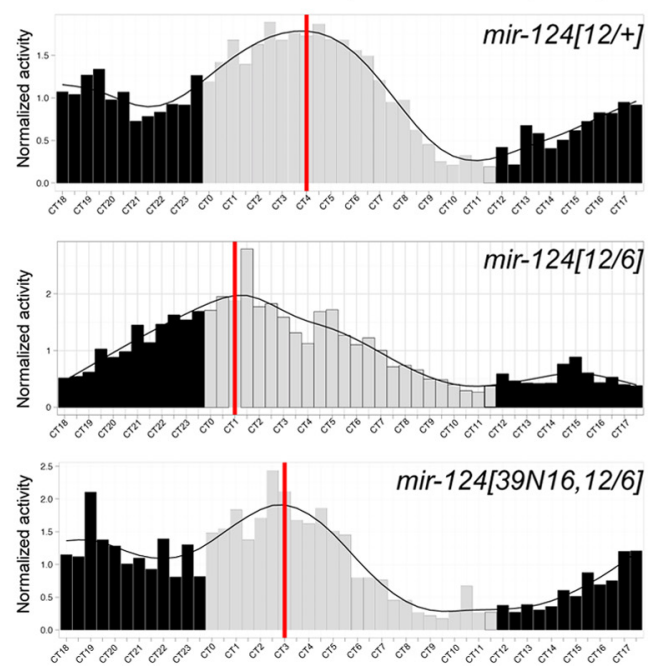

B

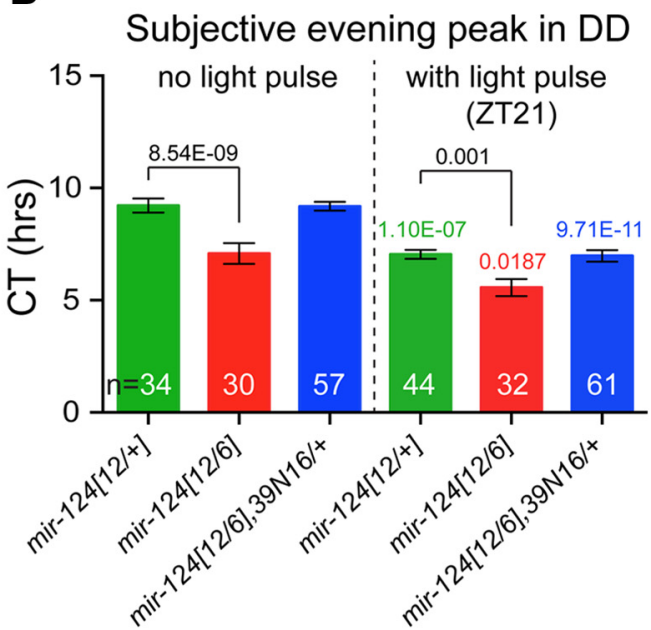

D Subjective evening peak in DD
with TC
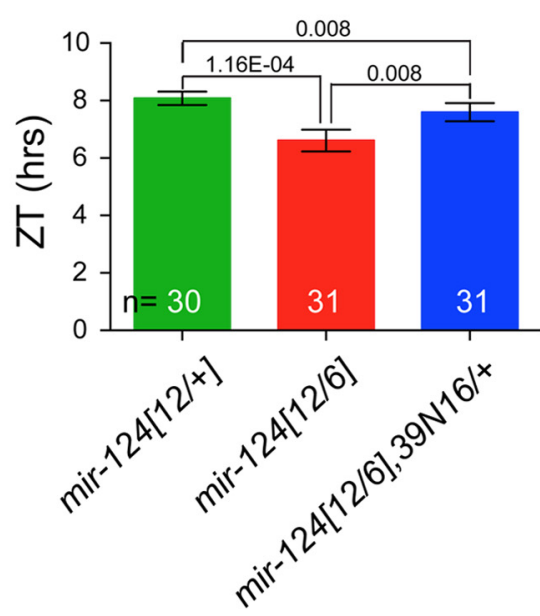

$\mathbf{F}$

Subjective evening peak in DD after TC
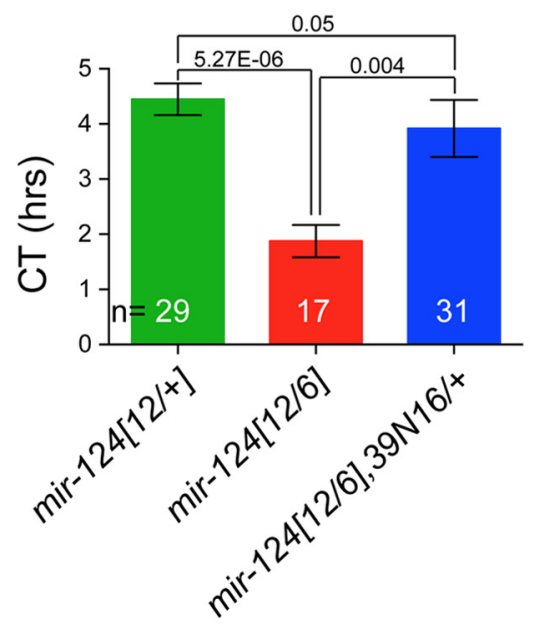

Figure 3. Behavioral responses of mir-124 mutants to environmental perturbations. $\boldsymbol{A}, \boldsymbol{B}$, Light-resetting analysis. $\boldsymbol{A}$, Following $L$ D entrainment, a $10^{\prime}$ light pulse was delivered at Zeitgeber time 21 (i.e., $3 \mathrm{~h}$ prematurely) before moving to DD (see Materials and Methods). The light pulse correspondingly shifts the evening peak in all genotypes analyzed. $\boldsymbol{B}$, Quantification of the subjective evening peak in the first $2 \mathrm{~d}$ following shift to DD conditions, without and with a premature light pulse. $\mathbf{C}, \mathbf{D}$, Analysis of temperature cycling in DD; black bars (Figure legend continues.) 
Immunohistochemistry. Adult heads were fixed for $3 \mathrm{~h}$ in $4 \%$ paraformaldehyde in $0.2 \%$ PBS containing $0.25 \%$ Triton X-100 (PBST). Heads were rinsed with $0.2 \%$ PBST three times and dissected in blocking solution ( $5 \%$ goat serum in $0.2 \%$ PBST). Primary and secondary antibodies were incubated overnight at room temperature. We used guinea pig-anti-Clockwork Orange (CWO; 1:200; Matsumoto et al., 2007), mouse-anti-PDF (1:1000; Developmental Studies Hybridoma Bank), goat-anti-PER (1:500; Santa Cruz Biotechnology sc-15720), rat-anti-TIM (1:1000; Young Laboratory), Alexa Fluor 488, 568, and 647 (1:500; Invitrogen). We analyzed PER and TIM staining in 5-10 neuronal clusters from 3-8 brains for each time point. Relative signals between genotypes were normalized to the median across each time series.

Behavioral assays and analyses. We assayed locomotor activities of healthy $<5 \mathrm{~d}$ males using the Drosophila Activity Monitor System (Trikinetics). For standard conditions, flies were maintained in $12 \mathrm{~h} \mathrm{LD}$ cycles at $25^{\circ} \mathrm{C}$. To analyze DD behavior, flies were entrained on $\mathrm{LD}$ cycles for $5 \mathrm{~d}$, then transferred to DD for $6 \mathrm{~d}$. For phase-shift experiments, we first entrained flies for five LD cycles. At Zeitgeber time 21 of the last day, animals were exposed to a 10 min light pulse, then transferred to DD. For temperature entrainment, flies were kept on $12 / 12 \mathrm{~h} 20 / 29^{\circ} \mathrm{C}$ thermocycle for $3 \mathrm{~d}$ in $\mathrm{DD}$, then moved to $25^{\circ} \mathrm{C}$.

For activity analyses, data were binned at $30 \mathrm{~min}$. For LD conditions, $5 \mathrm{~d}$ of data were used. For DD, we analyzed the first $2 \mathrm{~d}$ after transferring to darkness. Anticipation indices were calculated as the slope of activity changes from the last five (for morning anticipation) or eight (for evening anticipation) bins before light transitions, using the linear regression function in R. Circadian periods were calculated using ClockLab software (Actimetrics).

For statistical analysis of evening peak behavior times, we fitted daily fly behavioral profiles with a cubic smoothing spline (smoothing parameter, 0.6). Local maxima were called on smoothed curves for all individual flies, for each genotype. R packages "stats" and "quantmod" were used for curve fitting and peak calling (https://www.r-project.org/). The evening peak was defined by the highest activity before evening starts. Wilcoxon rank-sum tests, with multiple testing correction by Holm method, measured statistical significance of evening peaks between genotypes.

\section{Results}

\section{Deletion of mir-124 impairs diverse aspects of rhythmic locomotor behavior}

Having previously generated Drosophila mir-124 knock-outs (Sun et al., 2012), we examined their adult behaviors. We assayed male locomotion across $12 \mathrm{~h}$ LD cycles followed by DD, comparing heterozygous controls, trans-heterozygous mutants, and a 19 $\mathrm{kb}$ genomic rescue. We observed that mir-124 mutants exhibited a host of abnormal activity patterns under both LD and DD conditions and that these patterns were rescuable (Fig. $1 A-C$ ). We analyzed these in detail.

Under LD, control flies exhibit a bimodal behavior profile with increased activity before lights-on and lights-off transitions, known as morning and evening anticipations (Fig. 1D). Loss of mir-124 dramatically changed LD activities, with loss of morning anticipation and reduced evening anticipation (Fig. 1E). Con-

\section{$\leftarrow$}

(Figure legend continued.) indicate $20^{\circ} \mathrm{C}$; white bars indicate $29^{\circ} \mathrm{C}$. The evening activity peak of mir-124 mutants is still advanced under temperature cycling. $\boldsymbol{E}, \boldsymbol{F}$, Analysis of temperatureentrained animals, following release to constant conditions $\left(25^{\circ} \mathrm{C}\right)$. Note that the phase of all genotypes shifts, but mir-124 mutants exhibit greater phase advance. These assays use the same cohorts from $\boldsymbol{C}$ and $\boldsymbol{D}$, except dead animals were excluded from analysis; mir- 124 mutants have increased lethality following TC. $n=$ number of male animals analyzed. We used MannWhitney statistical tests for pairwise comparisons. For light-resetting analysis, we indicate significance values between controls and mutants in both conditions, and for each cognate genotype with and without light pulse (shown as color-coded $p$ values). Error bars indicate SEM. trols also exhibit an afternoon activity trough ("siesta"), which was less apparent in mutants. We noted specific defects in immediate responses to light transitions, which reflect startle behaviors. mir-124 mutants had normal lights-off response, but exhibited substantially reduced lights-on response (Fig. $1 A-C$, compare "morning" arrows, $E$ ). These LD phenotypes were rescuable (Fig. 1F).

We confirmed this using (1) additional mir-124 alleles from our laboratory, (2) an independently generated mir-124[177] knock-out (Weng and Cohen, 2012), and (3) hemizygotes over deficiency $D f(2 L)$ Exel7069. We observed similar behavioral defects in these diverse mir-124 combinations, and rescued independent backgrounds (Fig. 1G-I; data not shown). Therefore, mir-124 is causal for these behavioral defects. Moreover, while control female activity patterns are distinct from those of males, we observed mir-124 mutant females exhibited qualitatively similar changes in circadian behaviors as mir-124 males, and these were rescuable (data not shown). Therefore, overall mir-124 diurnal behavioral defects are not sexually dimorphic, as reported for its male-specific reproductive behavioral defects (Weng et al., 2013).

Following release into DD, wild-type flies maintain rhythmicity and bimodal activity patterns (Fig. $1 J$ ), although the amplitude of subjective morning peaks decays faster than subjective evening peaks. In contrast, mir-124 mutants exhibit unimodal behavior pattern in $\mathrm{DD}$, due to immediate loss of subjective morning activity (Fig. $1 B, K$ ). Strikingly, while loss of miR-124 does not affect DD period (Fig. $1 A-C$ ), it caused peak subjective evening activity to advance by several hours (Fig. $1 K$ ); this was especially apparent in the first several days after DD shift. Such activity phase phenotype is unusual among rhythmicity mutants, since advanced phase is usually accompanied by period change. These DD phenotypes were rescuable (Fig. $1 L$ ).

\section{Core activities of the circadian oscillator are functional in mir-124 mutants}

We attempted to narrow down the defect of mir-124 mutants. Their rhythmic phenotypes might involve defective core clock operation, altered clock output, or perhaps aberrant environmental perception.

We first considered whether miR-124 affected visual phototransduction. This was conceivable given mir-124 deletion impaired lights-on responses (Fig. 1). However, mir-124 mutants exhibited normal photoreceptor axonal projections to optic lobes (data not shown). We then used electroretinograms (ERGs) to investigate how mir-124 photoreceptors respond to light and transmit signals. Activity profiles were similar between control and two mir-124 mutants (Fig. 2A). We further analyzed photoreceptor responses to light stimulation by quantifying depolarization (Fig. $2 B$ ), and by measuring postsynaptic neurotransmission by quantifying "on" transients (Fig. 2C). Both parameters were unchanged in mir-124 mutants. Therefore, light perception, phototransduction, and synaptic transmission do not require miR-124.

Drosophila morning and evening activity peaks are attributed to discrete adult brain neurons: $\mathrm{M}$ and E cells (Grima et al., 2004; Stoleru et al., 2004). We analyzed expression of PDF and clockwork orange (CWO), which respectively label LNvs (including the s-LNvs or M cells) and all major circadian groups (including both $\mathrm{M}$ and E cells). Both LNv and LNd neurons expressed these markers normally in mir-124 mutant brains, and LNvs extended normal axonal and dendritic projections (Fig. 2D-I) and exhibited normal daily axonal morphological changes (Fernández et al., 2008; data not shown). 


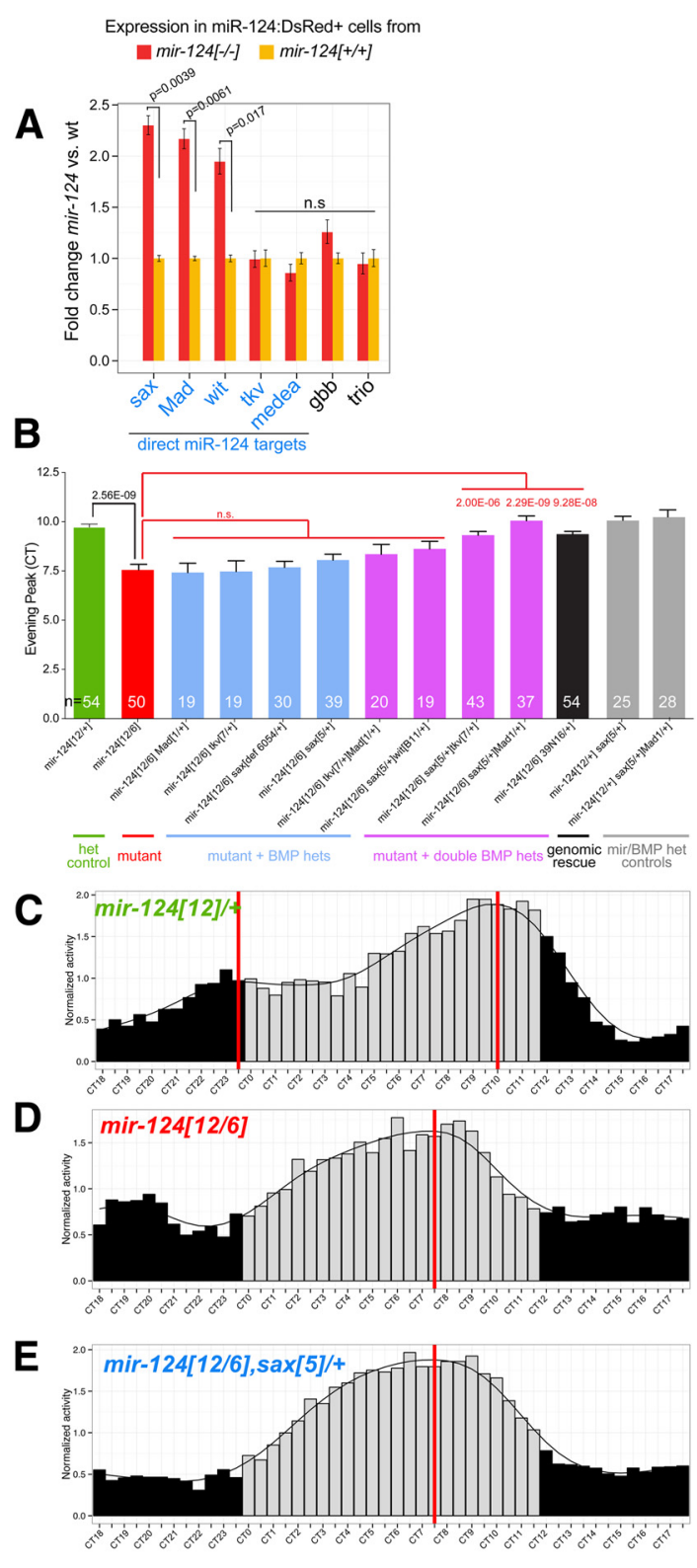

$\mathbf{F}$

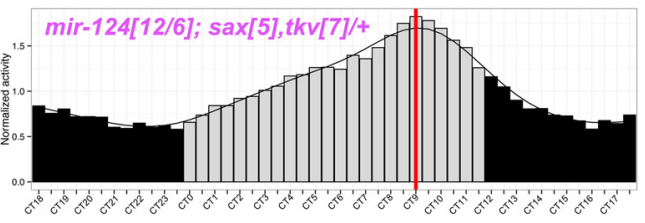

G

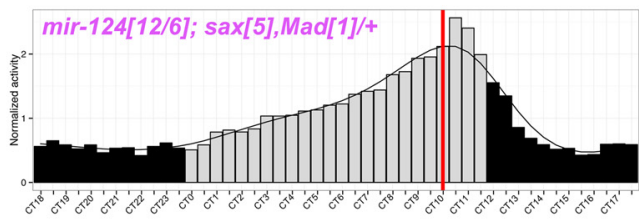

H

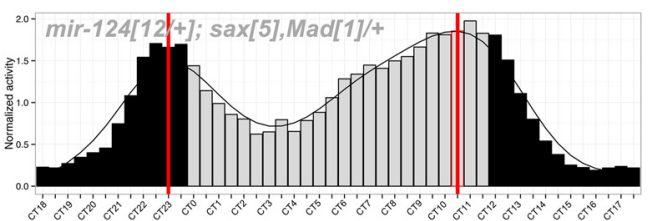

Figure 4. Suppression of specific mir-124 behavioral defects by BMP pathway mutants. $\boldsymbol{A}$, Derepression of multiple retrograde BMP pathway transcripts in mir-124 mutants. We reanalyzed triplicate array data from miR-124::DsRed-expressing cells of wild-type flies and of
Intrinsic clock operation during DD is maintained by PDFexpressing s-LNvs ( $\mathrm{M}$ cells). The normal period of mir-124 mutants implied that these clock cells functioned appropriately (Fig. 1). To assess this more directly, we stained for periodic nuclear accumulation of PER and TIM in DD. Figure $2 J, K$ shows comparable magnitude and phase of their oscillatory dynamics between mir-124 heterozygotes and homozygotes in s-LNvs. We extended this by quantifying PER and TIM cycling across multiple populations of core circadian neurons (Fig. $2 L$ ). As the molecular clock is functional in mir-124 mutants, this implies that miR-124 regulates clock output function.

\section{Responses of mir-124 mutants to environmental perturbations}

We probed the behavior of mir-124 mutants using other perturbations. First, we exploited a brief light pulse during late night to reset the clock, inducing phase advance. This is mediated by $\mathrm{M}$ and $\mathrm{E}$ cell functions (Lamba et al., 2014). We observed that mir124 mutants were receptive to light-mediated resetting. That is, a light pulse advanced the subjective evening peak of mir-124 mutants in DD conditions to the same extent as in control and rescued mutants (Fig. 3A,B). This provides further evidence for normal function of the core clock in these mutants.

Next, we noted that despite dampened activity patterns of mir-124 in LD, its activity phase in this condition was normal (Fig. 1E). This implied that light can set normal phase in mir-124 mutants. Since clocks can be synchronized by temperature, we tested whether temperature cycles (TCs) of $29^{\circ} \mathrm{C} / 20^{\circ} \mathrm{C}$ could restore phasing in the mutant. Under DD + TC conditions, mir-124 mutants exhibited detectable phase advance (Fig. $3 C, D$ ), a defect exaggerated following removal of temperature cues (Fig. $3 E, F$ ). Therefore, thermocycles did not fully compensate locomotor phase defects in mir-124 knock-outs.

\section{Reduction of BMP signaling suppresses specific mir-124 behavioral defects}

BMP signaling was recently implicated in clock regulation (Beckwith et al., 2013). Notably, we previously reported links between miR-124 and BMP signaling (Sun et al., 2012). Five core BMP components carry functional miR-124 sites in $3^{\prime} \mathrm{UTR}$, and three are derepressed at mRNA levels in mir-124 mutants (Fig. 4A). Moreover, mir-124 mutants exhibit aberrant synaptic transmission, phenocopying activated retrograde BMP signaling. We therefore tested for modification of mir-124 phenotypes by BMP pathway mutations. The rationale is that while miRNA mutants upregulate large target cohorts, as we showed with mir-124 mutant CNS (Sun et al., 2012), there can exist dose-sensitive targets whose modest reduction suppresses miRNA-related defects. This

mir-124 mutants (Sun et al., 2012). B, Quantification of the evening activity peak in various single and double heterozygous BMP mutant alleles in mir-124 mutants. The number of male flies analyzed per genotype is indicated. We used the Mann-Whitney statistical test for pairwise comparison of various data conditions. Compared with control mir-124 heterozygotes (green), mir-124 mutants exhibit an advanced activity phase. This is not modified by any single BMP heterozygote, or by several double BMP heterozygotes, but is significantly rescued by $s a x+t k v$ and sax + Mad double heterozygotes (purple), as it is by the mir-124 genomic transgene (black). Control mir-124/BMP heterozygotes are shown in gray. $\mathbf{C}-\boldsymbol{H}$, Examples of averaged DD activity profiles for various genotypes; subjective daytime hours are shaded gray. As shown in Figure 1, mir-124 mutants exhibit a unimodal evening activity peak with an advanced phase. The mir-124 phase is not modified by sax heterozygosity, but sax-tkv double heterozygotes partially rescue the phase and sax-Mad double heterozygotes restore this to the appropriate time. sax-Mad-mir-124 triple heterozygote, shown as a control, exhibits the normal bimodal pattern. Error bars indicate SEM in $\boldsymbol{A}$ and $\boldsymbol{B}$. 
scenario would implicate genes of particular phenotypic relevance to miRNA function.

We assayed several BMP pathway heterozygotes, including wit[B11], Mad[1], tkv[7], and sax[5], for modulation of mir124[12/6] phenotypes. We observed partial improvement of LD evening anticipation in some genotypes, although effects were modest (data not shown). We then turned to DD behavior, for which we documented unimodal behavior and advanced evening peak in mir-124 mutants (Fig. $4 B-D$ ). Neither phenotype was modified by any single BMP heterozygote (Fig. $4 B, E$ ), indicating they are not intrinsically easily modifiable. We then sensitized the system by testing double BMP heterozygotes. Among many combinations tested, double heterozygosity of sax with $t k v$ or Mad mutations substantially corrected the phase shift of mir-124 mutants (Fig. $4 B, F, G$ ). In these double BMP heterozygotes, mir-124 activity peaked toward the end of subjective daytime during DD, similar to the profile of normal animals. The mir-124 phase rescue was most robust in sax, Mad double heterozygotes (Fig. 4G), correlating with the fact these were the most strongly derepressed BMP components (Fig. 4A).

These results were not trivially due to dominant effects of BMP haploinsufficiencies, since various BMP single and double mutant heterozygotes in mir-124/+ backgrounds exhibited normal DD behavioral phasing (Fig. 4B, $H$ ). Moreover, we emphasize that phase was not corrected by the Zeitgeber temperature (Fig. 3C-F). Together, this extensive panel of genetic interaction tests yields evidence for functional contribution of deregulated BMP signaling to specific mir-124 defects in circadian behavioral output.

\section{Discussion}

As a well conserved neural miRNA, miR-124 is considered critical during vertebrate neural differentiation and human CNS disease (Sun et al., 2015a). However, extensive analyses of invertebrate mir-124 knock-outs showed it dispensable for gross neural development and differentiation (Clark et al., 2010; Sun et al., 2012; Weng and Cohen, 2012). Nevertheless, these mutants exhibit molecular and functional defects in the nervous system. Recently, miR-124 was found to control male reproductive behavior through repression of the splicing factor transformer (Weng et al., 2013). Our current studies uncover profound requirements for Drosophila miR-124 in functional outputs in the circadian timekeeping system, broadening the functional reach of this ancient neural miRNA. Similar conclusions were reached by an accompanying study (Zhang et al., 2016). Overall, the highly penetrant array of behavioral defects of mir-124 mutants contrasts with the typically modest defects of many miRNA mutants.

The phenotypes of mir-124 mutants reveal novel aspects of rhythmic biology. For example, we uncovered robust genetic suppression of DD phase advance in mir-124 mutants by mild reduction of BMP factors. Since we explicitly showed extensive direct and indirect expression changes in the mir-124 mutant CNS (Sun et al., 2012), it is conceivable other targets are responsible for different aspects of mir-124 aberrant behavior. Nevertheless, the functional relationship of miR-124 and BMP signaling is notable, especially as we showed miR-124 functions in clock output. BMP signaling adjusts the central oscillator to determine period (Beckwith et al., 2013), but its impact on phase determination was unknown. The former study misexpressed activated Sax + Tkv receptors to lengthen daily period, but mir-124 mutants exhibit normal period (Fig. 1). It is likely that forcing dual activated receptors using Gal4-UAS elevates BMP signaling much higher than does mir-124 deletion (Fig. 3A), so these ma- nipulations are not comparable. This illustrates how mir-124 mutants serve to identify new aspects of chronobiology.

Important questions await future resolution, including identities of neurons that are functionally affected in mir-124 mutants. Such knowledge is also necessary to approach how BMP signaling affects circadian behavioral output. Unfortunately, we were unable to rescue mir-124 behaviors using circadian drivers tim-Gal4, pdf-Gal4, or cry-Gal4 (data not shown). The binary system might induce inappropriate miR-124 levels, since panneural expression of miR-124 was lethal (data not shown). Alternatively, given evidence as a clock output regulator, miR-124 might function outside of core circadian neurons. Transgenic miRNA sponges have shown utility as competitive inhibitors (Fulga et al., 2015), but we could not phenocopy mir-124 mutants using pan-neural miR-124 sponge expression (data not shown). Thus, we could not use this strategy to map neurons to miR-124mediated behaviors. Somatic CRISPR (clustered regularly interspaced short palindromic repeat) technology may provide a means of cell-specific neuronal knock-outs (Shen et al., 2014) to advance this in the future.

Overall, mir-124-null mutants reveal new contributions of post-transcriptional regulation and signaling pathways to rhythmic behaviors. Moreover, the unique circadian output defects of mir-124 mutants, including the novel phase advance we characterized, make it an intriguing genetic probe for investigating changes in activity patterns in more complex environmental paradigms.

\section{References}

Allada R, Chung BY (2010) Circadian organization of behavior and physiology in Drosophila. Annu Rev Physiol 72:605-624. CrossRef Medline

Alvarez-Saavedra M, Antoun G, Yanagiya A, Oliva-Hernandez R, CornejoPalma D, Perez-Iratxeta C, Sonenberg N, Cheng HY (2011) miRNA-132 orchestrates chromatin remodeling and translational control of the circadian clock. Hum Mol Genet 20:731-751. CrossRef Medline

Beckwith EJ, Gorostiza EA, Berni J, Rezával C, Pérez-SantángeloA, Nadra AD, Ceriani MF (2013) Circadian period integrates network information through activation of the BMP signaling pathway. PLoS Biol 11:e1001733. CrossRef Medline

Busza A, Murad A, Emery P (2007) Interactions between circadian neurons control temperature synchronization of Drosophila behavior. J Neurosci 27:10722-10733. CrossRef Medline

Chen W, Liu Z, Li T, Zhang R, Xue Y, Zhong Y, Bai W, Zhou D, Zhao Z (2014) Regulation of Drosophila circadian rhythms by miRNA let-7 is mediated by a regulatory cycle. Nat Commun 5:5549. CrossRef Medline

Cheng HY, Papp JW, Varlamova O, Dziema H, Russell B, Curfman JP, Nakazawa T, Shimizu K, Okamura H, Impey S, Obrietan K (2007) microRNA modulation of circadian-clock period and entrainment. Neuron 54:813-829. CrossRef Medline

Clark AM, Goldstein LD, Tevlin M, Tavaré S, Shaham S, Miska EA (2010) The microRNA miR-124 controls gene expression in the sensory nervous system of Caenorhabditis elegans. Nucleic Acids Res 38: 3780-3793. CrossRef Medline

Fernández MP, Berni J, Ceriani MF (2008) Circadian remodeling of neuronal circuits involved in rhythmic behavior. PLoS Biol 6:e69. CrossRef Medline

Fulga TA, McNeill EM, Binari R, Yelick J, Blanche A, Booker M, Steinkraus BR, Schnall-Levin M, Zhao Y, DeLuca T, Bejarano F, Han Z, Lai EC, Wall DP, Perrimon N, Van Vactor D (2015) A transgenic resource for conditional competitive inhibition of conserved Drosophila microRNAs. Nat Commun 6:7279. CrossRef Medline

Grima B, Chélot E, Xia R, Rouyer F (2004) Morning and evening peaks of activity rely on different clock neurons of the Drosophila brain. Nature 431:869-873. CrossRef Medline

Helfrich-Förster C (2005) Neurobiology of the fruit fly's circadian clock. Genes Brain Behav 4:65-76. Medline

Kadener S, Menet JS, Sugino K, Horwich MD, Weissbein U, Nawathean P, Vagin VV, Zamore PD, Nelson SB, Rosbash M (2009) A role for mi- 
croRNAs in the Drosophila circadian clock. Genes Dev 23:2179-2191. CrossRef Medline

Lamba P, Bilodeau-Wentworth D, Emery P, Zhang Y (2014) Morning and evening oscillators cooperate to reset circadian behavior in response to light input. Cell Rep 7:601-608. CrossRef Medline

Lim C, Allada R (2013) Emerging roles for post-transcriptional regulation in circadian clocks. Nat Neurosci 16:1544-1550. CrossRef Medline

Luo W, Sehgal A (2012) Regulation of circadian behavioral output via a microRNA-JAK/STAT circuit. Cell 148:765-779. CrossRef Medline

Matsumoto A, Ukai-Tadenuma M, Yamada RG, Houl J, Uno KD, Kasukawa T, Dauwalder B, Itoh TQ, Takahashi K, Ueda R, Hardin PE, Tanimura T, Ueda HR (2007) A functional genomics strategy reveals clockwork orange as a transcriptional regulator in the Drosophila circadian clock. Genes Dev 21:1687-1700. CrossRef Medline

Shen Z, Zhang X, Chai Y, Zhu Z, Yi P, Feng G, Li W, Ou G (2014) Conditional knock-outs generated by engineered CRISPR-Cas9 endonuclease reveal the roles of coronin in C. elegans neural development. Dev Cell 30:625-636. CrossRef Medline

Stoleru D, Peng Y, Agosto J, Rosbash M (2004) Coupled oscillators control morning and evening locomotor behaviour of Drosophila. Nature 431: 862-868. CrossRef Medline

Stoleru D, Peng Y, Nawathean P, Rosbash M (2005) A resetting signal between Drosophila pacemakers synchronizes morning and evening activity. Nature 438:238-242. CrossRef Medline

Sun K, Westholm JO, Tsurudome K, Hagen JW, Lu Y, Kohwi M, Betel D, Gao
FB, Haghighi AP, Doe CQ, Lai EC (2012) Neurophysiological defects and neuronal gene deregulation in Drosophila mir-124 mutants. PLoS Genet 8:e1002515. CrossRef Medline

Sun Y, Luo ZM, Guo XM, Su DF, Liu X (2015a) An updated role of microRNA-124 in central nervous system disorders: a review. Front Cell Neurosci 9:193. CrossRef Medline

Sun K, Jee D, de Navas LF, Duan H, Lai EC (2015b) Multiple in vivo biological processes are mediated by functionally redundant activities of Drosophila mir-279 and mir-996. PLoS Genet 11:e1005245. CrossRef Medline

Vodala S, Pescatore S, Rodriguez J, Buescher M, Chen YW, Weng R, Cohen SM, Rosbash M (2012) The oscillating miRNA 959-964 cluster impacts Drosophila feeding time and other circadian outputs. Cell Metab 16: 601-612. CrossRef Medline

Weng R, Cohen SM (2012) Drosophila miR-124 regulates neuroblast proliferation through its target anachronism. Development 139:1427-1434. CrossRef Medline

Weng R, Chin JS, Yew JY, Bushati N, Cohen SM (2013) miR-124 controls male reproductive success in Drosophila. eLife 2:e00640. CrossRef Medline

Zhang Y, Emery P (2013) GW182 controls Drosophila circadian behavior and PDF-receptor signaling. Neuron 78:152-165. CrossRef Medline

Zhang Y, Lamba P, Guo P, Emery P (2016) miR-124 regulates the phase of Drosophila circadian locomotor behavior. J Neurosci 36:2007-2013. CrossRef Medline 